\title{
Different Shedding Patterns Measured by Virus Isolation and Real-Time PCR in Pigs Challenged with Aujeszky's Disease Virus
}

\author{
Vilmos Palya*, István Kiss, Tamás Mató, Zalán G. Homonnay, Balázs Felföldi and Edit Kovács \\ *Scientific Support and Investigation Directorate, Ceva-Phylaxia Veterinary Biologicals Co. Ltd, Budapest, Hungary
}

Received: : 08 May, 2017; Accepted: 25 May, 2017; Published: 04 June, 2017

*Corresponding authors: Vilmos Palya, Director of Scientific Support and Investigation Directorate, Ceva-Phylaxia Veterinary Biologicals Co. Ltd, Szállás utca, 1107, Budapest, Hungary, Tel: +361262 9505; Fax: +361260 3889; E-mail: vilmos.palya@ceva.com

\begin{abstract}
Aujeszky's Disease (AD) is a major pig disease that accounts for devastating economic losses to pig industry. AD is controlled by containment of infected herds, use of vaccines and removal of latently infected animals. In endemic areas, control programmes against $\mathrm{AD}$ rely on the use of vaccines that efficiently limit the replication of virulent virus in infected pigs. The presented study investigated the efficacy of a live vaccine, administered to the pigs by the intramuscular route in an oil/water emulsion adjuvant, to reduce the excretion of challenge virus by vaccinated pigs using virus isolation and real-time PCR (qPCR) to determine the amount of excreted virus.

Both virus detecting assays showed that the vaccinated animals shed significantly lower amount of virus than the nonvaccinated controls, and the quantity of the shed viruses decreased over time. Interestingly, while in the non-vaccinated control animals the virus titres detected by virus isolation and qPCR were approximately the same, in the vaccinated ones the amount of viable virus measured by virus isolation was consistently less than those detected by qPCR. These findings indirectly demonstrate that vaccination with a live virus induces effective local immunity at the primary site of replication, which reduces shedding and transmission of challenge virus. The results also pointed out that qPCR detection of the viral nucleic acid is not equal with viable viruses, which should be considered when interpreting the outcome of virus detecting assays.
\end{abstract}

Keywords: Aujeszky's disease; Vaccination; Virus shedding

\section{Introduction}

Aujeszky's disease, also known as pseudorabies, is caused by an alpha herpes virus that infects the central nervous system and other organs, such as the respiratory tract, in a variety of mammals except humans and the tailless apes [1]. Its natural host is the pig, which remain latently infected following clinical recovery, save piglets under 2 weeks old, which succumb to the disease due to encephalitis. The disease is controlled by containment of infected herds and by the use of vaccines and/or removal of latently infected animals [2].

Since the nasal mucosa is the primary site of AD Virus (ADV) infection and transmission of the virus is considered to occur mainly via the respiratory route, and it is important for the vaccines used in controlling the disease to induce a strong local immunity at that site, thus reducing the spread of ADV in a vaccinated pig population $[3,4]$.

The present study investigated how effectively a live $\mathrm{AD}$ vaccine can reduce challenge virus shedding by using two direct virus detecting methods: virus isolation and real-time PCR (qPCR).

\section{Materials and Methods}

Ten pigs, six-week-old white meat type and free from ADV and antibodies were vaccinated twice (four weeks apart) by the intramuscular (i.m.) route with the live $\mathrm{AD}$ vaccine, containing the attenuated, gE-deleted MNC+/10a ADV strain, in an oil/ water emulsion adjuvant (Auphyl Plus, Ceva-Phylaxia, Budapest, Hungary) [5]. Three weeks after booster vaccination the pigs were challenged with virulent ADV intranasally by using $4 \mathrm{ml}$ of 7.7 $\log 10$ TCID50/ml NIA-3 virulent strain of ADV. Five pigs served as un-vaccinated challenged controls; the challenge procedure was the same as for vaccinates. The pigs were observed for seven days for mortality, clinical signs, and relative body weight gain, i.e. body weight after the observation period divided by the body weight at challenge was calculated as a clinical measurement of vaccine efficacy [6]

Pigs in moribund stage were euthanized by overdosing sodium pentobarbital (Euthasol) according to the manufacturer's instructions. 
Before prime vaccination and challenge, blood samples were collected for Virus Neutralization (VN) assay using standard micro-neutralization test on MDBK cells against 100-300 TCID50 the vaccine strain [7].

In order to measure the amounts of excreted challenge virus nasal swabs (APTACA 0426) were taken from the left nostrils of the pigs on 2, 5, and 7 Days Post-Challenge (DPC). Swabs were washed in $1.5 \mathrm{ml}$ of MEM-H medium supplied with antibiotics, centrifuged at low speed $(1000 \mathrm{rpm})$ for $10 \mathrm{~min}$. The collected supernatants were serially diluted from $10^{1}$ to $10^{7}$ dilutions and then inoculated onto PK-15 cells and incubated for 6 days. After the incubation period, the TCID50 was determined according to Reed and Muench [8].

The same samples were further processed by qPCR capable of discriminating wild-type and gE-deleted vaccine viruses, having a lower sensitivity for detection of the gE gene [9]. Briefly, nucleic acid was extracted from the swabs by QIAxtractor nucleic acid purification device using DX Reagent Pack (Qiagen) according to the manufacturer's protocol. Quantity of ADV was determined with qPCR using the gB-specific primers and probe (labelled with FAM) as described by Ma et al. (2008). Results of the qPCR assay were analysed as Cq values. Moreover, the amount of virus measured by the qPCR in each sample was extrapolated to dilution series of a virus suspension with known titre analysed in parallel and expressed as TCID50/0.1mL $(\log 10$ TCID50/0.1mL).

Virus shedding data were analysed by one-way analysis of variance (ANOVA) using the Statgraphics software package. Differences were considered significant at $\mathrm{P}<0.05$.

\section{Results}

No clinical signs were observed in the negative control group throughout the observation period, while mucopurulent nasal discharge was observed among the non-vaccinated controls starting on 3 DPC. The condition of the controls deteriorated and three of them had to be euthanized due to their moribund stage on 6 DPC, and one on 7 DPC post challenge.

All pigs were seronegative against ADV at the time of vaccination. Before challenge, the vaccinated group of animals had a mean of $8.46 \log 2 \mathrm{VN}$ titer while the control animals were seronegative for antibodies to ADV. Following challenge the mean value of body temperature of the vaccinated group increased by less than $0.5^{\circ} \mathrm{C}$ by $2 \mathrm{DPC}$, then returned to normal, while the un-vaccinated group showed fever (up to $41.5^{\circ} \mathrm{C}$ from the $39.5^{\circ} \mathrm{C}$ baseline value) throughout the observation period. The mean relative body weight gain was 1.11 and 0.86 for the vaccinated and the control group, respectively.

Both virus detecting assays resulted in significantly lower amount of virus shedding by the vaccinated animals than by the non-vaccinated controls at each sampling date (Figure 1). Challenge virus shedding in the vaccinated pigs was three and two orders of magnitude less as detected by virus isolation and $\mathrm{gB}$ targeting qPCR, respectively. In the vaccinated group, higher titres were measured consistently by qPCR than by virus isolation, while in the control group, higher virus titres were measured by virus isolation than by qPCR, except in the surviving single control pig on 7 DPC. By the end of the observation period eight out of the ten vaccinated pigs stopped shedding infectious $A D V$, although seven were still qPCR positive.

There was agreement between the results of the qPCRs targeting gB or gE, as demonstrated by the individual measurements on 5 DPC and alike the data published on the used protocol (Figure 2) [9].

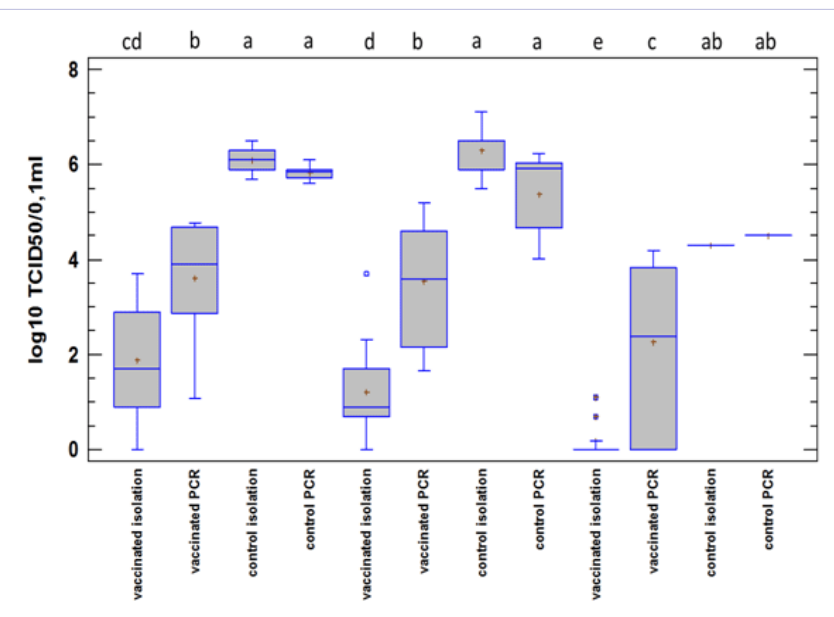

Figure 1: Box-and-Whisker Plot of challenge ADV shedding data upon vaccination as measured by virus isolation and quantitative real-time PCR targeting the gB gene of ADV; DPC means days post challenge. Different letters mean statistically significant differences between group means converted to $\log 10$ TCID $50 / 0.1 \mathrm{ml}$

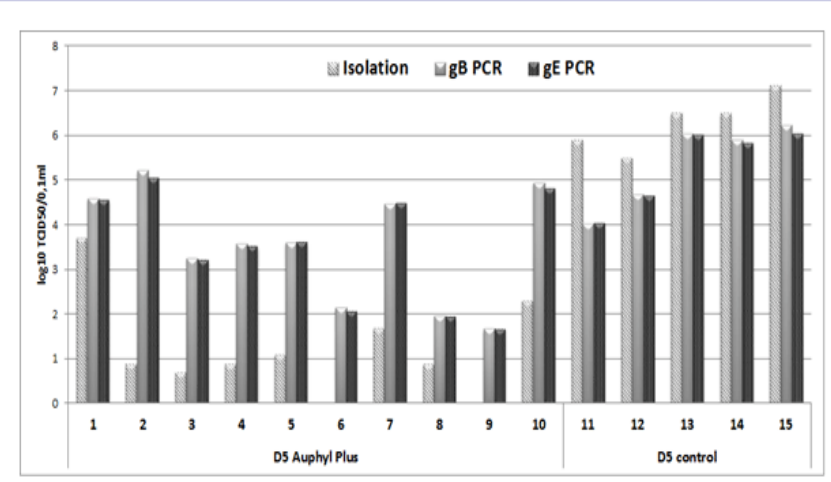

Figure 2: Individual virus shedding data of vaccinated and non-vaccinated animals on the fifth day after challenge with Aujeszky's disease virus.

\section{Discussion}

The clinical manifestation of Aujeszky's disease can successfully be controlled by vaccination [10]. However, vaccination cannot prevent infection, especially with larger quantities of $\mathrm{ADV}$, which subsequently establish latent infection in the trigeminal ganglion and central nervous system. The virus then can be reactivated (even by transport-induced stress, for 
example) and excreted via nasal and pharyngeal fluids [11]. Thus, in the epizootiology of $\mathrm{AD}$ these latently infected pigs have key importance for spreading the virus. Therefore, vaccination aims at reducing virus excretion as much as possible.

The efficacy of Auphyl Plus based on protection against weight loss, clinical signs and increase of body temperature was reported [12]. The present study investigated the vaccine induced reduction of challenge virus excretion by means of two analytical approaches, i.e. virus isolation and qPCR. Vaccinated animals shed significantly lower amount of virus after challenge and for a shorter period of time than the non-vaccinated controls, i.e. three orders of magnitude less viable, thus, potentially transmissible virus. Interestingly, the titres of ADV shed by the non-vaccinated control animals proved to be higher as measured by virus isolation than by qPCR. This was consistent in each pig, except one pig that survived till the end of the observation period 7 DPC, when higher amount of shed virus could be detected by qPCR than by virus isolation. In contrast, in the vaccinated pigs this trend was the opposite, i.e., higher amount of shed virus was detected by qPCR than by virus isolation. In the frame of this trial it was not possible to seek for explanation to this observation.

The replication kinetics and characteristics of ADV in the respiratory tract are influenced by several factors, such as virus strain, inoculation route, virus titre in the inoculum, animal age, genetics and immune status $[13,14]$. It is well documented that vaccination induces resistance against virus growth in the oronasal mucosa, consequently reduces the excretion of challenge virus shedding; however, vaccines greatly differ in this respect [15-20]. Cellular immune responses were associated with the differing kinetics between the virus excretion of vaccinated and non-vaccinated pigs $[4,21,22]$. The lower amount of viable virus detected by virus isolation than those detected by qPCR in the vaccinated animals reflects the effect of a local immune response through cytotoxic $\mathrm{T}$ cells and interferon production, which reduce ADV replication and shedding [22,23]. At the same time, no neutralizing activity of the nasal washings was found after ADV challenge [24]. Nevertheless, the ADV-specific antibodies plausibly also contribute to the clearance of virus, by inducing antibody-dependent cell-mediated cytotoxicity or viral uptake by macrophages [25]. To better understand the mechanisms behind these findings more sensitive methods are needed to enable direct in vivo analysis of the immune responses of pigs.

Bouma et al. demonstrated that intranasal ADV infection resulted in a considerable inflow of various cell subpopulations (in particular, CD8+ T-cell subsets) to the site of infection, both in unvaccinated and vaccinated pigs, which might play important role in clearance of ADV [4]. The increase in density of the CD4+ and CD8+ cell subsets, however, occurred earlier in the tissues of vaccinated than in the unvaccinated pigs.

Our findings are in good agreement with the above findings, as demonstrated that (i) in pigs vaccinated with a live $A D$ vaccine the effect of (supposedly cell-mediated) immune response significantly reduced the amount of viable ADV shedding compared to non-vaccinated control animals, and (ii) in the controls a similar effect, i.e., reducing the amount of ADV that could be propagated below that measured by qPCR, was delayed until 7 DPC post challenge, reflecting a difference in kinetics of the primary response in unvaccinated pigs and the secondary immune response in vaccinated pigs [4]. Since the secondary immune response occurred in the presence of ADVspecific antibodies, which can neutralize the virus, this could have contributed to the reduced live virus load of the vaccinated animals.

Conclusively, our study demonstrated the distinct kinetics of local immune mechanisms by means of the quantitative analysis of total and viable virus excretion. The finding were also remarkable from data interpretation point of view, in particular for virus transmission related studies, i.e., the demonstration of the viral nucleic acid does not necessarily mean the presence of viable/transmittable viruses.

\section{Acknowledgments.}

Edit Fodor's contribution to virus isolation and neutralization assays is acknowledged.

\section{References}

1. Mettenleiter TC. Aujeszky's disease (pseudorabies) virus: the virus and molecular pathogenesis--state of the art, June 1999. Vet Res. 2000;31(1):99-115. doi: 10.1051/vetres:2000110

2. Wittmann G. Aujeszky's disease. Scientific and Technical Review of the Office International des Epizooties 1986;5(4):959-977

3. Wittmann G, Jakubik J, Ahl R. Multiplication and distribution of Aujeszky's disease (pseudorabies) virus in vaccinated and nonvaccinated pigs after intranasal infection. Archives of Virology. 1980; 66(3):227-240

4. Bouma A, Zwart RJ, De Bruin MG, De Jong MC, Kimman TG, Bianchi AT. Immunohistological characterization of the local cellular response directed against pseudorabies virus in pigs. Vet Microbiol. 1997;58(24):145-154. doi: 10.1016/S0378-1135(97)00168-5

5. Lomniczi B, Wehmann E. A genetically improved Aujeszky's disease vaccine strain derived from $\mathrm{K} / 61$ displays enhanced antigenicity in pigs. Hungarian Veterinary Journal. 1998;120(4):195-203

6. Anonymous: Aujeszky's disease vaccine (live) for pigs for parenteral administration. 2014:0745. Edited by 9.0. PE; 2014

7. OIE. Aujeszky's disease. In: Manual of Diagnostic Tests and Vaccines for Terrestrial Animals 2016. edn.; 2016;97-113

8. Reed LJ, Muench H. A simple method of estimating fifty per cent endpoints. Am J Hyg. 1938;27(3):493-497. doi: 10.1093/ oxfordjournals.aje.a118408

9. Ma W, Lager KM, Richt JA, Stoffregen WC, Zhou F, Yoon KJ. Development of real-time polymerase chain reaction assays for rapid detection and differentiation of wild-type pseudorabies and gene-deleted vaccine viruses. J Vet Diagn Invest. 2008;20(4):440-447. doi: $10.1177 / 104063870802000405$

10. Freuling CM, Muller TF, Mettenleiter TC. Vaccines against pseudorabies 
virus (PrV). Vet Microbiol. 2016. doi: 10.1016/j.vetmic.2016.11.019

11. Wittmann G. Aujeszky's disease: factors important for epizootiology and control. Scientific and Technical Review of the Office International des Epizooties. 1985;4(1):5-20

12. Lomniczi B, Kelemen M. Efficacy of live Aujeszky-vaccine containing strain $\mathrm{MNC}+10 \mathrm{~A}$ in pigs. Hungarian Veterinary Journal. 1998;120(9):515-522

13. Nauwynck H, Glorieux S, Favoreel H, Pensaert M. Cell biological and molecular characteristics of pseudorabies virus infections in cell cultures and in pigs with emphasis on the respiratory tract. Vet Res. 2007;38(2):229-241. doi: 10.1051/vetres:200661

14. Pol JM, Gielkens AL, van Oirschot JT. Comparative pathogenesis of three strains of pseudorabies virus in pigs. Microb Pathog. 1989;7(5):361-371

15. Labarque GG, Nauwynck HJ, Maes DG, Pensaert MB. Protection of fattening pigs against challenge with Aujeszky's disease virus after a successive intranasal/intramuscular vaccination. Vet Q. 1999;21(3):104-107. doi: 10.1080/01652176.1999.9695003

16. Lomniczi B. Efficacy of vaccines and vaccination against Aujeszky's disease of pigs. I. Experiments with live vaccines in seronegative (susceptible) pigs. Hungarian Veterinary Journal. 1990;45(12):735741

17. Lomniczi B. Efficacy of vaccines and vaccinations against Aujeszky's disease of pigs. II. Experiments with inactivated vaccines in seronegative pigs. Hungarian Veterinary Journal. 1991;46(2):81-86

18. Pensaert MB, De Smet K, De Waele K. Extent and duration of virulent virus excretion upon challenge of pigs vaccinated with different glycoprotein-deleted Aujeszky's disease vaccines. Vet Microbiol. 1990;22(2-3):107-117
19. Vannier P, Hutet E, Bourgueil E, Cariolet R. Level of virulent virus excreted by infected pigs previously vaccinated with different glycoprotein deleted Aujeszky's disease vaccines. Vet Microbiol. 1991;29(3-4):213-223

20. Vannier P, Hutet E, Cariolet R. Influence of passive immunity on pig immunization with deleted Aujeszky's disease vaccines measured by the amount of wild virus excretion after challenge. Vet Microbiol. 1995;43(1):53-63

21. Kimman TG, De Bruin TM, Voermans JJ, Peeters BP, Bianchi AT. Development and antigen specificity of the lymphoproliferation responses of pigs to pseudorabies virus: dichotomy between secondary B- and T-cell responses. Immunology. 1995;86(3):372-378

22. van Rooij EM, de Bruin MG, de Visser YE, Middel WG, Boersma WJ, Bianchi AT. Vaccine-induced T cell-mediated immunity plays a critical role in early protection against pseudorabies virus (suid herpes virus type 1) infection in pigs. Vet Immunol Immunopathol. 2004;99(12):113-125. doi: 10.1016/j.vetimm.2004.01.004

23. Nauwynck HJ, Pensaert MB. Cell-free and cell-associated viremia in pigs after oronasal infection with Aujeszky's disease virus. Vet Microbiol. 1995;43(4):307-314

24. Martin S, Wardley RC. Local humoral and cellular responses in Aujeszky's disease virus infection in pigs. Res Vet Sci. 1987;42(2):170174

25. Kensinger M, Eskew ML, Scheuchenzuber W, Zarkower A. Porcine effector mechanisms: antibody-dependent cell-mediated cytotoxicity of pseudorabies-infected target cells. Vet Immunol Immunopathol. 1987;14(3):223-231 